
proteins, some of which are found in fimbriae; and pertactin (or 69-kD protein), a well-characterized nonfimbrial agglutinogen. 


\section{THE ADVENT OF RECOMBINANT PERTUSSIS VACCINES}

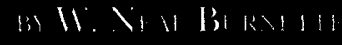

SIALIC ACID GLYCOCONJUGATE RECOGNITION SITE he pertussis ingredient of the diphtheria-tetanus-pertussis (DTP) vaccine has long been known to cause a high rate of mild-to-moderate side effects in infants. Whether it is responsible, as some have implied, for acute encephalopathy, irreversible brain damage, and even death, remains controversial. Even though epidemiologic data suggest that the relationship between DTP vaccination and catastrophic neurologic events of infancy may be purely temporal, this has not lessened the public demand for a less-reactive pertussis vaccine. The biotechnology industries, in collaboration with government and academic laboratories, have responded by using recombinant DNA methods to produce defined pertussis components for such vaccines. Significant industrial research contributions have come from Amgen (Thousand Oaks, CA), Connaught Centre for Biotechnology Research (Willowdale, Ontario, Canada), Sclavo Research Centre (Siena, Italy), and Wellcome Research Laboratories (Beckenham, Kent, U.K.).

\section{Pertussis: Disease and Vaccine}

Pertussis, or whooping cough, is a particularly devastating respiratory infection in infants and young children. In addition to the morbidity and mortality associated with bronchopneumonia and atelectasis, the paroxysmal coughing spasms so characteristic of the disease can themselves lead to permanent neurologic impairment at a rate approximately 33 times greater than has ever been attributed to the vaccine by its worst critics. Following a long term of development and extensive clinical trials, a pertussis vaccine was widely introduced in the United States in the late $1940 \mathrm{~s}$. It is composed of chemically-treated or heat-inactivated bacterial cells of Bordetella pertussis, the causative agent of the disease. Albeit not as elegant as the isolated and inactivated toxins (known as toxoids) of diphtheria and tetanus, the wholecell pertussis constituent of the DTP vaccine proved strikingly effective in preventing infant whooping cough wherever it was employed. In the United States, for example, the incidence of disease dropped from a reported high of 157 cases per 100,000 in 1934 to 1.4 per 100,000 in 1988. But 20-60 million children in developing countries still suffer from this disease each

W. Neal Burnette is a research scientist at Amgen, 1900 Oak Terrace Lane, Thousand Oaks, CA 91320. 
year, not as a result of an inadequate vaccine but as a general consequence of the abject failure of the industrialized world to reach the susceptible global population with protective immunizations.

\section{Pertussis Toxins and Antigens}

In recent years, attempts have been made to fractionate $B$. pertussis with the intention of separating immunoprotective antigens from reactogenic components (Figure 1). This work has been frustrated by the absence of serologic correlates of protective immunity. Researchers still disagree about the extent to which the known bacterial antigens contribute to protective responses. Indeed, it is not known whether protection is mediated by humoral or cell-mediated immunity, or both. Nevertheless, clinical trials of "acellular" pertussis vaccines have indicated that the major protein exotoxin, commonly called pertussis toxin (PTX), is important for the prevention of severe disease. It has been proposed that other $B$. pertussis components may confer immunoprotection, perhaps by eliciting antibodies that abrogate bacterial attachment to the respiratory epithelium and preclude subsequent colonization. In this regard, investigators have lately been examining such outer membrane components as filamentous hemagglutinin (FHA), a $69 \mathrm{kD}$ protein ("pertactin"), and various serotypic agglutinogens (Figure 1).

Pertussis toxin, in addition to being a presumptively vital protective immunogen, is also a major component in bacterial pathogenesis and a likely contributing agent to the adverse reactions provoked by pertussis vaccine. PTX is an extraordinarily active pharmacologic substance with biological effects that have given it a variety of names: islet-activating protein, histamine-sensitizing factor, and lymphocytosis-promoting factor. Moreover, it is a potent adjuvant and an adhesin that likely contributes to bacterial attachment. With the exception of this latter characteristic, the activities of PTX appear to derive from its catalytic properties. It is an ADP-ribosyltransferase that covalently modifies certain guaninenucleotide binding $(\mathrm{G})$ proteins of the eukaryotic cell membrane involved in the transduction of hormonal signals. Stringent inactivation of PTX-containing vaccines, whether whole-cell or acellular, destroys its biological activities and thereby abolishes its ability to elicit vaccine reactions. Such strenuous treatment, however, has a deleterious effect on its known protective antigenic determinants. On the other hand, less exhaustive treatment preserves these immunogenic epitopes, but permits the toxin to retain reactivity. It is also important to note that chemical or heat treatment of pertussis vaccine materials may be vital for the inactivation of the other protein toxins produced by $B$. pertussis: adenylate cyclase and dermonecrotic toxin.

\section{Recombinant Pertussis Toxin}

PTX had previously been shown to possess five distinct protein subunits organized into a hexameric quaternary structure (Figure 2) comprised of an A protomer (S1 subunit) and a B oligomer (subunits S2, S3, S4, and S5). Although it was recognized that the $S 1$ subunit possessed the catalytic properties and likewise accommodated the dominant mouse protective epitope of the toxin, the potential immunoprotective contributions made by the $B$ oligomer were not known. The molecular cloning and sequence determination of the PTX operon (Locht and Keith, 1986. Science 232:1258) offered the opportunity to explore structure-function relationships in the toxin and perhaps divine a means to genetically inactivate the pharmacologic activities of the molecule without losing its immunogenic attributes. The approach taken by our laboratory was to produce each of the subunits in Escherichia coli (thus eliminating the possibility of contamination by other $B$. pertussis toxic components), define regions of the $S 1$ subunit contributing to enzyme activity and formation of the dominant protective epitopes, utilize site-specific mutagenesis to eliminate the former without affecting the latter, and finally to assemble the holotoxin molecule in vitro from the recombinant subunits to create a genetic holotoxoid free from the other reactive components of $B$. pertussis.

We expressed the genes encoding the various PTX subunits to high levels in E. coli (Burnette et al., 1988. Bio/Technol ogy 6:699) and found that recombinant S1 retained the ADP-ribosyltransferase activity of the native molecule as well as reactivity with a monoclonal antibody (1B7) that defined the dominant mouse-protective antigenic determinant. This subunit was nonetheless incapable of eliciting a protective response in mice, suggesting that the epitope might require a constrained conformation induced by association of S1 with the B oligomer. Progressive terminal deletion of the $\mathrm{Sl}$ gene followed by expression of truncated S1 polypeptides in $E$. coli indicated that a domain bounded by tyrosine- 8 and proline-15 was crucial for enzyme activity and formation of the protective epitope (Cieplak et al., 1988. Proc. Natl. Acad. Sci. USA 85:4667). Perhaps not surprisingly, this region is one of a number in PTX that shares significant amino acid sequence homology with the A subunits of Vibrio cholerae toxin and $E$. coli heat-labile enterotoxin, both of which are also ADPribosyltransferases.

\section{Site-SPECIFIC MUTAGENESIS AND THE Genetic TOXOID}

The congruence of functions within such a limited region of the $S 1$ subunit presented a problem in protein engineering. How was the virulence-related enzymatic activity of the S1 subunit to be eliminated while preserving both its epitope and its potential to acquire the secondary structure essential for association with the $\mathrm{B}$ oligomer subunits? This problem was confronted by producing a series of recombinant analog S1 polypeptides in which selective amino acid substitutions were made in the eight-residue tract (tyrosine- 8 to proline-15) by site-specific codon mutagenesis of the S1 gene (Burnette et al., 1988. Science 242:72). A single mutation-the substitution of lysine for arginine-9-possessed the requisite characteristics of antigenic reactivity and catalytic inactivity. Whereas subsequent investigations have revealed the participation of other regions of the S1 subunit in substrate recognition and catalysis, none has manifested such an absolute requirement as arginine- 9 .

But did this $S 1$ analog retain a conformation that permitted holotoxin formation? To address this question, recombinant $S 1$ of authentic amino acid sequence $(\mathrm{rS} 1 / 1)$ and the analog ( $\mathrm{rS} 1 / 1-4)$ were each purified, permitted to fold under controlled conditions, and allowed to individually associate in vitro with native B oligomer (Bartley et al., 1989. Proc. Natl. Acad. Sci. USA 86:8353). In both cases, holotoxin species that comigrated in gel electrophoresis with native PTX could be clearly identified. Further, the enzymatically active rSl/1-containing holotoxin caused a quantitative cytopathic response in cultured Chinese hamster ovary cells, indistinguishable from that of native toxin, while the analogcontaining holotoxin species lacked any substantial cytopathicity. These results confirmed that the pathogenicity of the toxin resides in the enzyme activity of its S1 moiety, and that a potentially nonreactogenic "holotoxoid" could be created by recombinant DNA technology. Preliminary animal experiments with the genetic toxoid (J.L. Arciniega and D.L. Burns, pers. comm.) further indicate the absence of in vivo biological activity, as measured by its failure to stimulate leukocytosis and histamine sensitization.

\section{Pertussis Holotoxoid, Other RECOMBINANT ANTIGENS, AND VACCINE DEVELOPMENT}

Investigations directed at obtaining a holotoxoid exclusively from $E$. coli-produced PTX subunits have continued. Formation of B oligomer from recombinant $S 2, S 3, S 4$, and $S 5$ has been achieved with relatively high efficiency, and its association with recombinant SI has also 
been attained, albeit with significantly diminished yield (unpublished results). Recently, we have begun molecular engineering of the $\mathrm{B}$ oligomer, particularly the $\mathrm{S} 2$ and $\mathrm{S} 3$ subunits, with the aim of modifying its cellular recognition domains and further dissipating the biological activities of the toxin.

Another current approach to manufacturing a recombinant pertussis holotoxoid has been demonstrated by researchers at Sclavo (Pizza et al., 1989. Science 246:497) and Connaught (Zealey et al. Bio/Technology, this issue). These groups have introduced inactivating mutations into the PTX S1 subunit by deleting the TOX operon in $B$, pertussis and subsequently substituting site-specific mutant analogs of the operon by homologous recombination. The advantage of this method is that the authentic host organism is capable of assembling and secreting the holotoxoid species. In these studies, a series of S1-containing analogs of PTX were obtained with various single and double substitutions at arginine-9, arginine-13, arginine- 58 , and glutamate129. Both studies concluded that the most biochemically and biologically inactive analog contained the double mutation of lysine-9/glycine-129. The Sclavo team also showed that this mutant retained good reactivity with the mouse-protective monoclonal antibody 1B7. Human clinical safety trials have subsequently been initiated with this candidate toxoid vaccine. A potential disadvantage of manufacturing recombinant vaccine materials in B. pertussis is that they will be suspected of containing contaminants of the other toxins known to be produced by this human pathogen (see above). For this reason, regulatory authorities may insist they be chemically inactivated, as are other accllular vaccine components prepared from $B$. pertussis, thus negating the recombinant benefits of genetic inactivation coupled with unmodified immunogenicity.

Certain other components found in the organism may also elicit protective immune responses, augmenting anti-PTX immunoprotection, and perhaps prevent bacterial colonization. The latter type of immunity, if it can be stimulated in older children and adults, may be essential for precluding transmission to infants and could eventually result in cradication of pertussis. In this respect, much current research has focused on outcr membrane proteins of B. pertussis, such as FHA and pertactin. The latter protein, antibodies against which can be found in high titers in children immunized with certain acellular vaccines, was recently shown to provide solid protection in mice against the effects of acrosol challenge with $B$. pertussis (Shahin et al., 1990. J. Exp. Med. 171:63). Now researchers from Wellcome (Makoff ct al. Bio/Technology, this issuc) have shown that this immunogen can also be synthesized in significant amounts by recombinant $l$. coli, making it feasible to evaluate its clinical potential for prevention of pertussis either alone or in combination with recombinant PTX holotoxoid.

\section{Conclusions}

In just four years since the PTX operon was cloned, we have gained insights into structure-function relationships of this complex molecule and of the other pertussis components that would have been unattainable without the tools of molecular genetics. Continued investment in this research and the application of rational protein design and engincering will inevitably result in a defined recombinant DNA-derived vaccine with reduced side-effects and the potential to abolish a disease that, to this day, kills nearly one child in every fifty born on the planet.

For a free copy of this article (while available). write in 501 on Reader Service Card

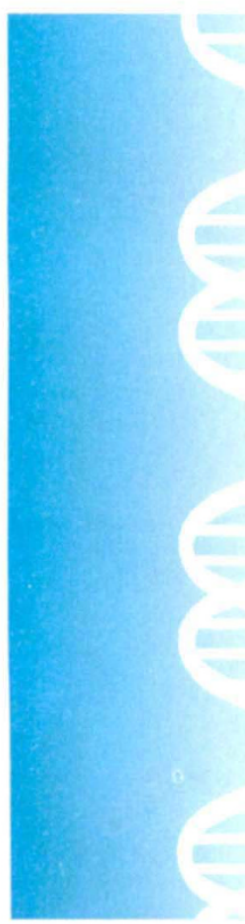

BIOPHARM Heidelberg

\section{From Contract Research...}

\author{
DNA Cloning \\ Expression in Pro- and Eucaryotic Cells \\ Sequencing of DNA \\ Purification and Characterization of Proteins \\ Analysis of Carbohydrates \\ Determination of Contaminants via PCR, ELISA etc.
}

Validated Standard Operating Procedures

Documentation for Registration

\section{...To Analytical Services}

\author{
Czernyring 22 \\ D-6900 Heidelberg 1 \\ Phone: + $49622114024-26$ \\ Fax: + 496221163574
}

